\title{
FRAM-The Robotic Telescope for the Monitoring of the Wavelength Dependence of the Extinction: Description of Hardware, Data Analysis, and Results
}

\author{
Michael Prouza, ${ }^{1}$ Martin Jelínek, ${ }^{2}$ Petr Kubánek, ${ }^{2,3}$ Jan Ebr, ${ }^{1}$ \\ Petr Trávníček, ${ }^{1}$ and Radomír Šmída ${ }^{1}$ \\ ${ }^{1}$ Institute of Physics, Academy of Sciences of the Czech Republic, 18221 Praha 8, Czech Republic \\ ${ }^{2}$ Instituto de Astrofísica de Andalucía (IAA-CSIC), 18008 Granada, Spain \\ ${ }^{3}$ GACE, Image Processing Laboratory, University of Valencia, 6980 Paterna (Valencia), Spain
}

Correspondence should be addressed to Michael Prouza,prouza@fzu.cz

Received 24 August 2009; Accepted 2 December 2009

Academic Editor: Alberto J. Castro-Tirado

Copyright ( 2010 Michael Prouza et al. This is an open access article distributed under the Creative Commons Attribution License, which permits unrestricted use, distribution, and reproduction in any medium, provided the original work is properly cited.

\begin{abstract}
FRAM- F/(Ph)otometric Robotic Atmospheric Monitor is one of the atmospheric monitoring instruments at the Pierre Auger Observatory in Argentina. FRAM is an optical telescope equipped with CCD cameras and photometer, and it automatically observes a set of selected standard stars. Primarily, FRAM observations are used to obtain the wavelength dependence of the light extinction. FRAM telescope is also able to observe secondary astronomical targets, and namely the detection of optical counterparts of gamma-ray bursts has already proven to be successful. Finally, a wide-field CCD camera of FRAM can be used for rapid monitoring of atmospheric conditions along the track of particularly interesting cosmic ray showers. The hardware setup of the telescope, its software system, data taking procedures, and results of analysis are described in this paper.
\end{abstract}

\section{Introduction}

The FRAM telescope is a part of the Pierre Auger Observatory $(\mathrm{PAO})[1]$, which is currently the largest detector of the ultra-high energy cosmic rays (ultra-high energy cosmic rays (UHECRs) are defined as having their energy higher than $10^{18} \mathrm{eV}$.). The finished southern site of the PAO is located in Mendoza province in Argentina, close to the Andes mountain range, and in the vicinity of the town Malargüe $\left(69^{\circ} \mathrm{W}, 35^{\circ} \mathrm{S}, 1400 \mathrm{~m}\right.$ a.s.l. $)$.

For the correct determination of the properties of the cosmic ray showers, it is necessary to have precise knowledge about the immediate status of the atmosphere. Therefore, there is a whole range of different atmospheric monitoring instruments at the PAO. The main task of the FRAM telescope is the continuous monitoring of the wavelength dependence of the atmospheric extinction. FRAM works as an independent, RTS2-driven [2], fully robotic system, and it performs a photometric calibration of the sky on various UV-to-optical wavelengths using a $0.2 \mathrm{~m}$ telescope and a photoelectric photometer. As a primary objective, FRAM observes a set of chosen standard stars. From these observations, it obtains instant extinction coefficients and the extinction wavelength dependence. The instrument was installed during 2005 and after some optimizations it is routinely taking data since June 2006.

The main advantage of the system is a fast measurement-data for one star in all filters are usually obtained in less than five minutes. In comparison to Central Laser Facility (CLF) (the CLF is a laser with calibrated output, located approximately in the center of the detector array. The CLF automatically fires a set of shots every 15 minutes, and it is being observed by all the fluorescence detector telescopes. The analysis of the CLF signal is then used to determine the actual atmospheric conditions.) [3] or lidars (four lidars (a name originally derived from LIght Detection And Ranging) are currently in operation at the Pierre Auger Observatory, each one is situated close to the one of four fluorescence detector buildings. Lidar shoots calibrated laser signal in the air and then 


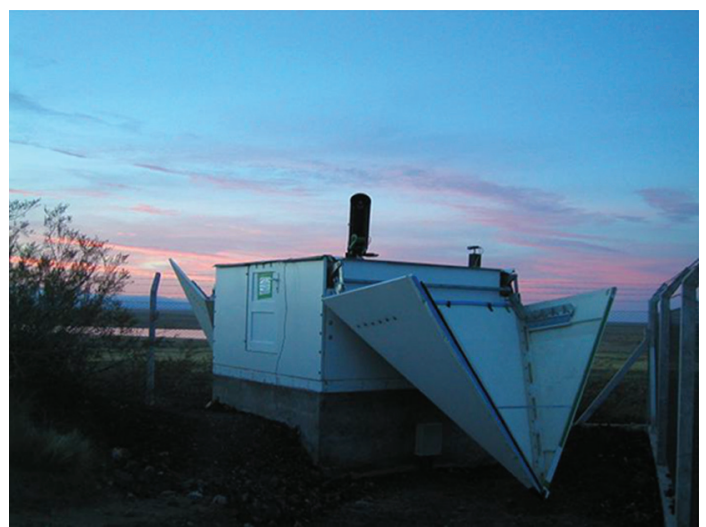

FIGURE 1: Original configuration FRAM telescope and its enclosure during sunset at the Los Leones site.

observes the backscattered signal with high-time resolution. From the analysis of the intensity of this returned signal it is then possible to determine the amount of scatterers (and specifically of aerosols) in different heights above the ground.) [4], the other advantage of FRAM is that its measurements are completely non-invasive, that is, producing no light that could affect the data acquisition of the fluorescence detector (FD). Furthermore, some of the stars are well calibrated and nonvariable standard light sources with precisely known and tabulated intensities in various filters, thus allowing straightforward comparison with measurements done using the same set of filters. On the other hand, the main disadvantage of this instrument is its limited capability of only integral measurements through the whole atmosphere.

\section{Instrument Setup}

The telescope has its own enclosure made of laminated glass fiber epoxy with inner iron frame. The enclosure is located about 30 meters from the building of the fluorescence detector at Los Leones (see Figure 1), on the southern edge of the Pierre Auger Observatory array and about 13 kilometers from the town of Malargüe in Mendoza province in Argentina $\left(69^{\circ} \mathrm{W}, 35^{\circ}\right.$, altitude $\left.1400 \mathrm{~m}\right)$.

2.1. Original Setup. As a primary instrument, we used a $20 \mathrm{~cm}$ Cassegrain-type telescope with an Optec SSP-5A photometer and with integrated 6-position filter slider. Effective telescope focal length was $2970 \mathrm{~mm}$ and focal ratio $\sim 1: 15$. The system was further equipped with an electronic focuser Optec TCF-S. This Crayford-style motorized focuser was installed in secondary Cassegrain focus and the photometer was attached to its moving end. A beam-splitting dichroic mirror was installed behind the focuser. The red and infrared light was reflected into narrow-field pointing CCD camera (Starlight XPress MX716) and ultraviolet and visible light passed through the mirror into the photometer.

Narrow-field pointing CCD camera had a resolution of $752 \times 580$ pixels and field of view of $7^{\prime} \times 5^{\prime}$. It was primarily
TABLE 1: FRAM filter characteristics.

\begin{tabular}{lccc}
\hline $\begin{array}{l}\text { Filter } \\
\text { position }\end{array}$ & $\begin{array}{c}\text { Filter } \\
\text { name }\end{array}$ & $\begin{array}{c}\lambda_{\text {centre }} \\
(\mathrm{nm})\end{array}$ & $\begin{array}{c}\text { Filter } \\
\text { FWHM }(\mathrm{nm})\end{array}$ \\
\hline 0 & Dark & - & - \\
1 & $U$ & 360 & 70 \\
2 & $u$ & 350 & 38 \\
3 & $v$ & 411 & 19 \\
4 & $b$ & 467 & 18 \\
5 & $y$ & 547 & 23 \\
\hline
\end{tabular}

used for the fine centering of the targeted star into a field of view of the photometer, which had only $1^{\prime}$ in diameter.

Photometer Optec SSP-5A is a high-precision stellar single channel photometer. A Fabry lens projects an image of the primary mirror onto the cathode of photomultiplier (PMT). The Hamamatsu R6358 PMT was selected for our setup, because of extended spectral response from $185 \mathrm{~nm}$ to $830 \mathrm{~nm}$. A Fabry lens is of B270-type glass that has enhanced UV-transmission. This still somewhat cuts down the transmission below $350 \mathrm{~nm}$, but does not adversely affect the transmission of any of the used filters. For star measurements, we use the set of four Strømgren uvby filters and Johnson $U$ filter (see Table 1 for filter characteristics).

Atop the telescope was installed wide-field CCD camera-Finger Lake Instrumentation (FLI) MaxCam CM8 with Carl Zeiss Sonnar $200 \mathrm{~mm} \mathrm{f/2.8} \mathrm{telephoto} \mathrm{lens.} \mathrm{This}$ CCD camera uses Kodak KAF 1603 ME chip with $1536 \times$ 1024 pixels, thus assuring $240^{\prime} \times 160^{\prime}$ field of view. The effective diameter of the lens is $57 \mathrm{~mm}$ and the limiting magnitude under optimum conditions reached $R \sim 15.0$ for a 30-seconds exposure. This wide-field CCD camera was primarily dedicated for astrometry. A median astrometric error for bright objects (signal to noise ratio > 7) and 120second exposure is about $0.5^{\prime \prime}$ (i.e., about $1 / 20$ of WF camera pixel).

The mount was a commercially available Losmandy G11, which used the standard GEMINI GOTO system equipped with two servomotors with relative optical encoders.

2.2. Upgrade in Summer 2009. The FRAM system was significantly upgraded in July 2009. The mount was replaced with Software Bisque Paramount ME German equatorial mount, that is, equipped with MKS 4000 dual-axis DC servomotor control system. The telescope was replaced with the commercially available $12^{\prime \prime}$ Meade LX200 SchmidtCassegrain telescope.

Furthermore, the pointing narrow-field CCD camera was replaced for a second FLI MaxCam CM8 camera $(1536 \times 1024$ pixels), and the dichroic mirror was replaced with the small prism (off-axis guider optical element) that reflects part of the light from the telescope to the photometer, but which is not obscuring the field of view of the narrow-field camera. Therefore, both photometer and narrow field camera now receive full spectra of the light that is being observed by the telescope. 
Finally, the wide-field camera was equipped with the FLI filter wheel CFW-2 with the set of Johnson-Coussins UBVRI filters. This will allow us to perfome comparative studies of the wavelength dependence of the extinction from photometric and wide-field CCD measurements.

Due the presence of the corrector plate in the new Schmidt-Cassegrain telescope, the new setup has very constrained spectral sensitivity below $400 \mathrm{~nm}$. It is questionable if we will be able to produce any good-quality results with photometer using Johnson $U$ or Strømgren $u$ filters (see also Table 1).

Currently (August 2009), the new setup is still in its comissioning stage, and no observation results are available. All the results presented in Section 6 were obtained with the original telescope setup.

\section{Software}

The system is driven by RTS2, or Remote Telescope System, 2nd Version, software package [2]. RTS2 is an integrated package for remote telescope control under the Linux operating system. It is designed to run in fully autonomous mode, selecting targets from a database table, storing CCD image and photometer metadata to the database, processing images and storing their identified coordinates in the database. RTS2 was developed and is maintained under open-source license in collaboration with robotic telescope projects of BART, BOOTES, and WATCHER [5].

\section{Observed Targets and Observation Schedule}

FRAM is primarily designed to provide the atmospheric extinction model. The data for this model are collected by the photometer with the help of both CCD cameras. The observation targets are selected bright (brighter than $6.5 \mathrm{mag}$ ) standard stars from the photometric catalogue of Perry et al. [6] that features star measurements in the Strømgren uvby photometric system [7]. Currently, we are using about 25 stars in the selection that are located at suitable declinations and homogenously distributed over the right ascension.

The target cycle begins with a slew to the position followed by a short WF camera exposure to check the pointing accuracy. The position of the photometer aperture within WF camera's image is well known, so if the initial pointing is not satisfactory, a correction could be made. This image also serves as a test of atmospheric conditions: target may be canceled, if the necessary conditions are not met (clouds or fog resulting in no image astrometry).

After the star of interest was successfully centred within the WF aperture, a control exposure with the NF CCD camera is done. The star is identified as the brightest source in the field of view and, if needed, the mount position is corrected again and the star is moved to the centre of photometer aperture. The photometer then does two sequences of measurements per filter of interest. Each sequence typically consists of five 1-second integrations to obtain the signal value and its variance in each filter.
Simultaneously, both CCD cameras take exposures, so that pointing may be improved in real-time. The WF camera provides also a measurement in set of Johnson-Coussins UBVRI filters. The complete set of measurements is then stored in the structure of PSQL database.

\section{Optical Follow-Ups of Gamma-Ray Bursts}

The RTS2 software system was originally developed especially for the search of optical transients of gamma-ray bursts. This software system was significantly modified to achieve FRAM main aims in atmospheric monitoring; however, it is still very easy to activate special observation mode for optical transients. The main computer of the system receives in such case the alerts about detected gamma-ray bursts via network, slews there and makes images of the given sky region.

This alert system was activated on FRAM in late 2005, and already during January 2006 a very successful observation was made. An extraordinarily bright prompt optical emission of the GRB 060117 was discovered and observed with a wide-field CCD camera atop the telescope FRAM from 2 to 10 minutes after GRB. Optical counterpart identified in our images was characterized by rapid temporal flux decay with slope exponent $\alpha \propto 1.7 \pm 0.1$ and with a peak brightness of $10.1 \mathrm{mag}$ in Bessel $R$ filter. Later observations by other instruments set a strong limit on the optical and radio transient fluxes, unveiling an unexpectedly rapid further decay. We presented more details in [8].

\section{Calibration and Results}

Our main goal is to provide the so-called Angstrom exponent $\gamma$, which is often used for parametrization of wavelength $(\lambda)$ dependence of aerosol optical depth $\tau_{A}: \tau_{A}(\lambda)=\tau_{A 0} \cdot\left(\lambda_{0} / \lambda\right)^{\gamma}$, where $\lambda_{0}$ is the reference wavelength and $\tau_{A 0}$ is the aerosol optical depth measured for this wavelength [9]. Moreover, the Johnson $U$ filter has almost the same central wavelength as have the lasers used for measurement of vertical aerosol optical depth (VAOD) at CLF [3] and at lidar stations [4]. The integral value of VAOD $(h=\infty)$ in $U$ filter thus can be used for direct cross-checks with these instruments.

We analyzed our database of photometer counts since June 2006, when the telescope achieved a stable mode of operation, until December 2008.

Using the available analysis of the CLF data, we initially selected a set of very clear ("Rayleigh") nights, when no aerosols (or only negligible amounts of aerosols) were observed by the CLF instrument. We used these data for calibration of FRAM photometer. Under the assumption of presence of no aerosols, we fitted the dependence of difference of observed and tabulated magnitudes on airmass. The resulting dependence should be linear and the fit parameters characterize both the extinction and the instrument properties. The slope for clear nights should be in agreement with the computed expectations for the Rayleigh scattering, and the value of the intercept defines the conversion of the instrumental magnitude. The knowledge of the real observed magnitudes then allowed us to directly compute the 


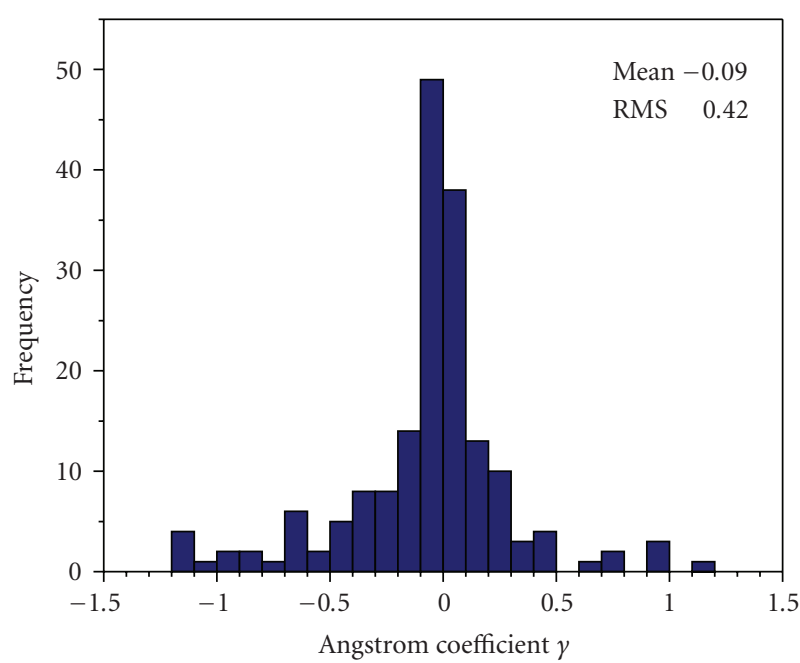

(a)

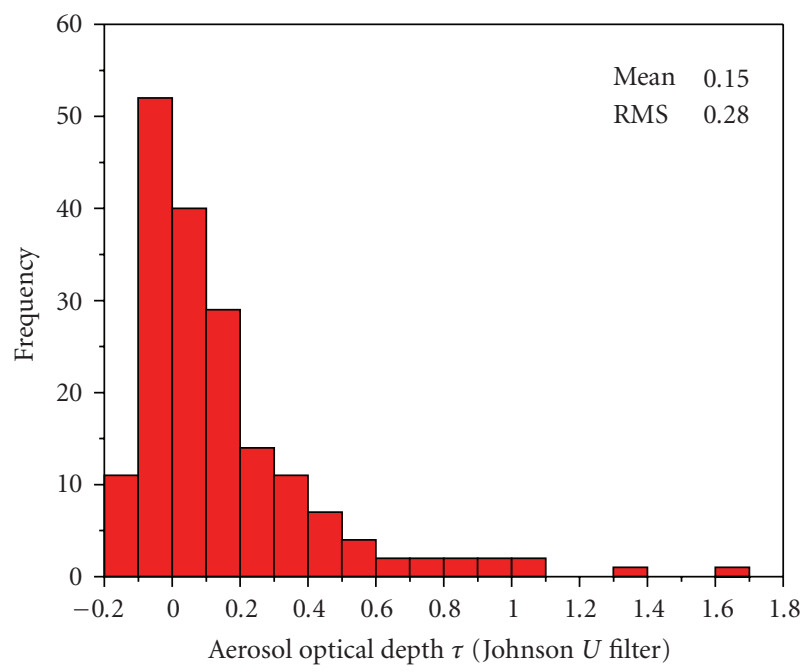

(b)

Figure 2: Histograms of measured distribution of Angstrom coefficient $\gamma$ (a) and of integral aerosol optical depth $\tau$ (b) for all data obtained from June 2006 until December 2008. Quality cuts from wide-field camera and photometer were applied as described in the text.

extinctions for our whole database, including all other (i.e., non-Rayleigh) nights. After that, we converted extinction expressed in magnitudes into total optical depth and then subtracted molecular Rayleigh part, using model from [10].

For the analysis of the aerosol extinction wavelength dependence, we used only-high quality data. For our selection, we required that three following conditions were fulfilled simultaneously. First, the limiting magnitude of the wide-field camera images was higher than 13 (clear sky). Second, the fluctuations in the number of detected stars in the wide-field camera images were smaller than $30 \%$ between individual images. The third cut is then on the fluctuations of the photometer readings. If the fluctuations were higher than square root of the signal (e.g., due to strong wind), the measurement was also not used.
For the standard star measurements (see Figure 2), we obtained a preliminary mean value of $\gamma=-0.1 \pm 0.4$ that is, lower than the results from HAM instrument $(\gamma=0.7 \pm 0.5)$ [11]; however, $1 \sigma$ limits of both instruments are overlapping.

Moreover, FRAM $\gamma=-0.1$ is in good agreement with theoretical expectations for the atmosphere in desert-like environment of the Argentinian pampa $(\gamma \sim 0)$ [12], where coarse-grain particles prevail.

\section{Acknowledgments}

The authors from the Institute of Physics in Prague (M. Prouza, J. Ebr, P. Trávníček and R. Šmída) would like to acknowledge the support from the Academy of Sciences of the Czech Republic (AV0Z10100502 and AV0Z10100522) and from the Ministry of Education, Youth and Sports of the Czech Republic (MSMT-CR; projects LA08016, LC527, 1M06002, and MSM0021620859). The collaborators from Spain (M. Jelínek and P. Kubánek) were supported by the Grant of the Spanish Ministry of Science and Innovation (AYA 2007-063677). Finally, all authors acknowledge the ARAE group at IAA-CSIC for its support.

\section{References}

[1] J. Abraham, P. Abreu, M. Aglietta, et al., "Operations of and future plans for the Pierre Auger observatory," in Proceedings of the 31st International Cosmic Ray Conference (ICRC '09), Lodz, Poland, July 2009.

[2] P. Kubánek, M. Jelínek, S. Vítek, et al., "RTS2: a powerful robotic observatory manager," in Advanced Software and Control for Astronomy, vol. 6274 of Proceedings of SPIE, Orlando, Fla, USA, May 2006.

[3] B. Fick, M. Malek, J. A. J. Matthews, et al., "The central laser facility at the Pierre Auger observatory," Journal of Instrumentation, vol. 1, no. 1, Article ID P11003, 17 pages, 2006.

[4] S. Y. BenZvi, R. Cester, M. Chiosso, et al., "The Lidar system of the Pierre Auger observatory," Nuclear Instruments and Methods in Physics Research, Section A, vol. 574, no. 1, pp. 171-184, 2007.

[5] P. Kubánek, M. Jelínek, S. Vítek, et al., "Status of robotics telescopes driven by RTS2 (BART, BOOTES, FRAM and Watcher)," Nuovo Cimento B, vol. 121, no. 12, pp. 1501-1502, 2006.

[6] C. L. Perry, E. H. Olsen, and D. L. Crawford, "A catalog of bright UVBY beta standard stars," Publications of the Astronomical Society of the Pacific, vol. 99, pp. 1184-1200, 1987.

[7] B. Strömgren, "Two-dimensional spectral classification of F stars through photoelectric photometry with interference filters," Vistas in Astronomy, vol. 2, pp. 1336-1346, 1956.

[8] M. Jelínek, M. Prouza, P. Kubánek, et al., "The bright optical flash from GRB 060117," Astronomy and Astrophysics, vol. 454, no. 3, pp. L119-L122, 2006.

[9] J. Abraham, P. Abreu, M. Aglietta, et al., "Upper limit on the cosmic-ray photon fraction at EeV energies from the Pierre Auger observatory," Astroparticle Physics, vol. 31, no. 6, pp. 399-406, 2009.

[10] A. Bucholtz, "Rayleigh-scattering calculations for the terrestrial atmosphere," Applied Optics, vol. 34, no. 15, pp. 2765-2773, 1995.

[11] S. Y. BenZvi, M. Bohacova, B. Connolly, et al., "New method for atmospheric calibration at the Pierre Auger observatory 
using FRAM, a robotic astronomical telescope," in Proceedings of the 30th International Cosmic Ray Conference (ICRC '07), Merida, Mexico, July 2007.

[12] T. F. Eck, B. N. Holben, J. S. Reid, et al., "Wavelength dependence of the optical depth of biomass burning, urban, and desert dust aerosols," Journal of Geophysical Research D, vol. 104, no. 24, pp. 31333-31349, 1999. 

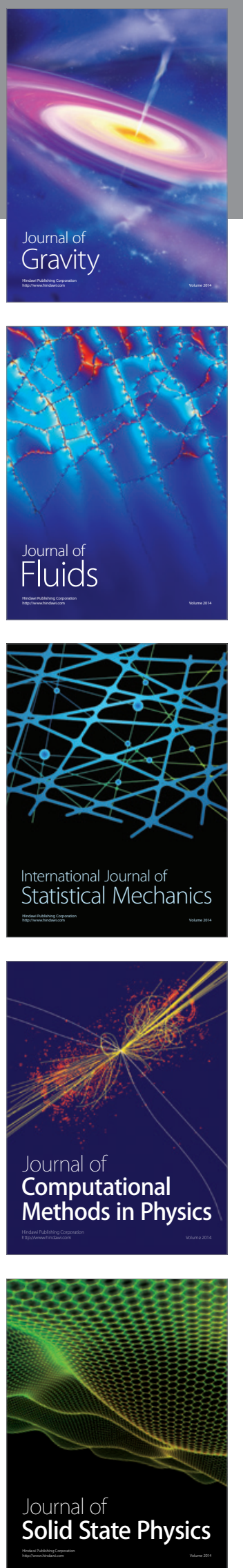

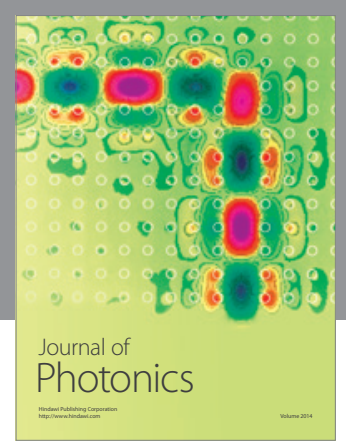

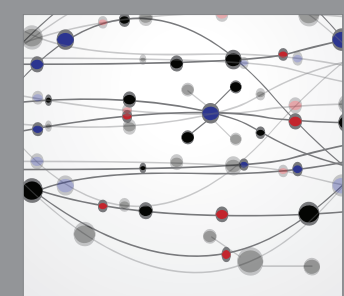

The Scientific World Journal
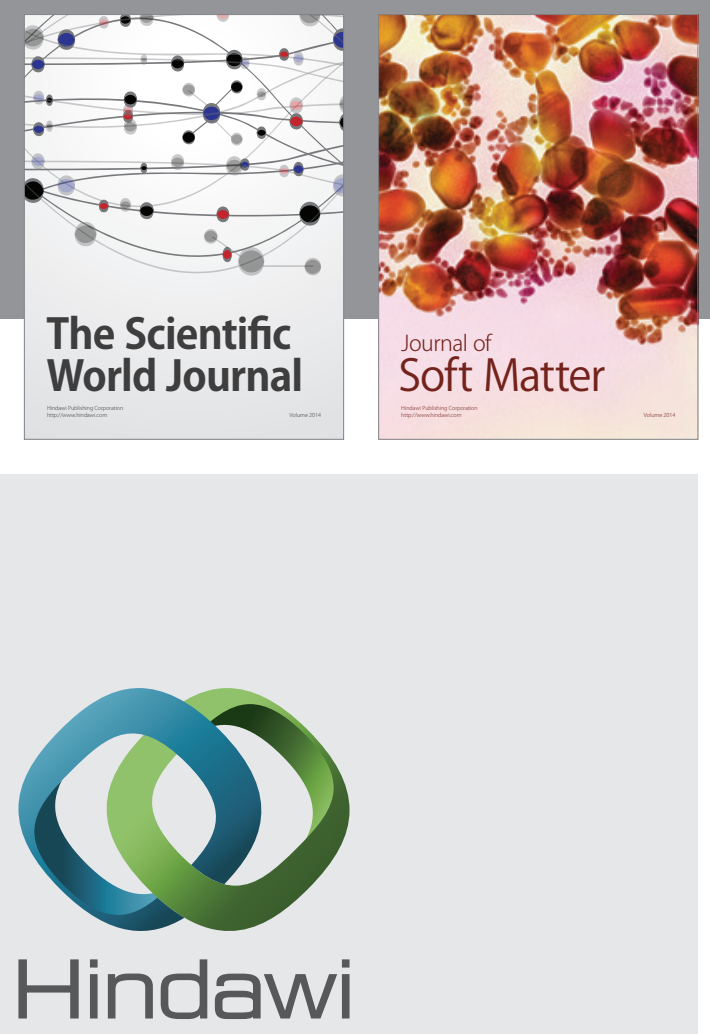

Submit your manuscripts at

http://www.hindawi.com
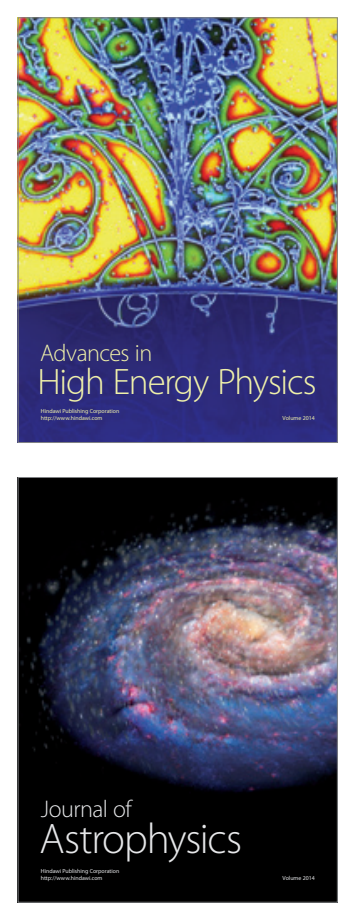
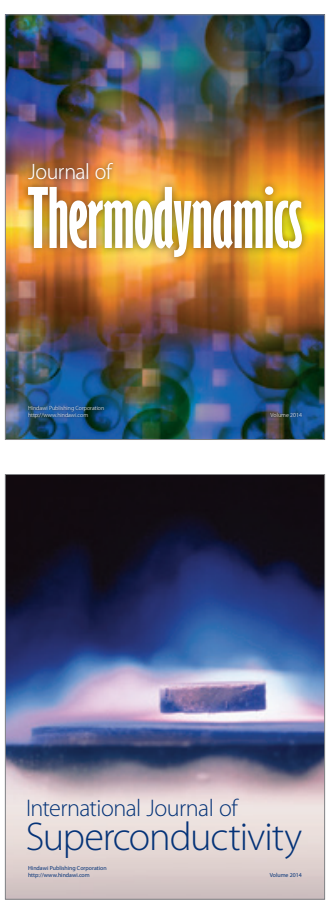
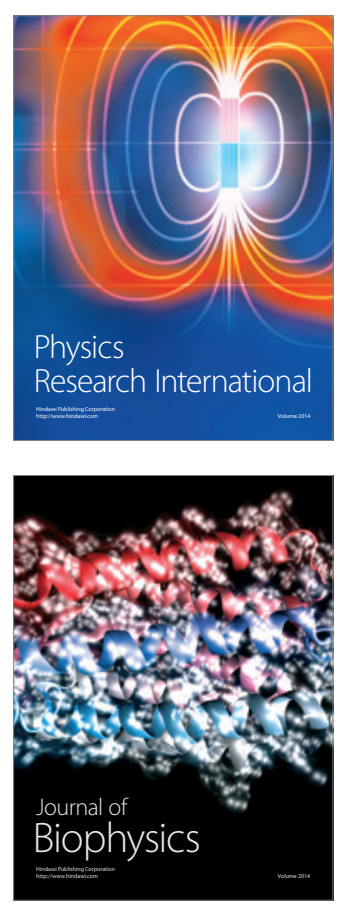
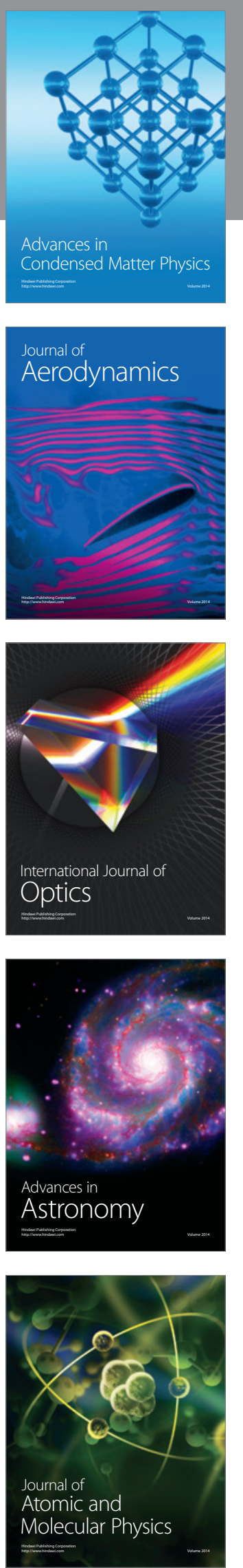